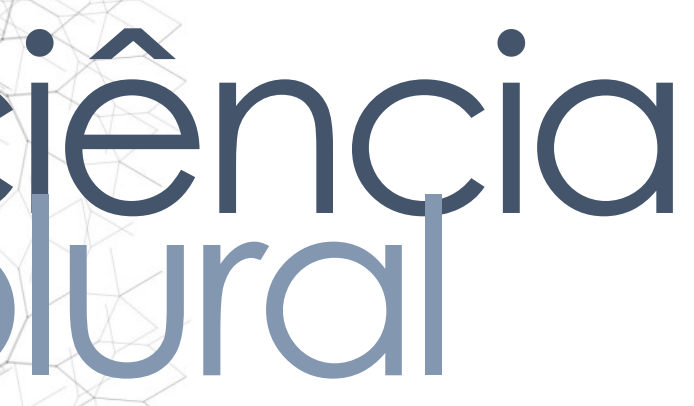

\title{
A EDUCAÇÃO EM SAÚDE NO PROCESSO DE TRABALHO DOS PROFISSIONAIS DA ESTRATÉGIA SAÚDE DA FAMÍLIA: RELATO DE EXPERIÊNCIA
}

Health education in the work process of Family Health Strategy professionals: experience report

Educación em salud en el proceso de trabajo de profesionales de la Estrategia de Salud Familiar: informe de experiencia

Antônia Fernanda Sousa de Brito • Enfermeira • Especialista em Saúde da Família • Residente em Saúde Coletiva na Escola de Saúde Pública do Ceará • E-mail: nanda.fernanda18@hotmail.com

Ciliane Macena Sousa • Enfermeira • Especialista em Saúde da Família, Gestão e Saúde e em Urgência, Emergência e UTI • Enfermeira-Intensivista e Emergencista no Hospital São Luiz - Mossoró-RN • E-mail: cily_macena@hotmail.com

Autora responsável pela correspondencia:

Antônia Fernanda Sousa de Brito • E-mail: nanda.fernanda18@hotmail.com 


\section{RESUMO}

Introdução: A Estratégia Saúde da Família é constituída por equipes multiprofissionais que devem atuar na perspectiva interdisciplinar, articulando suas práticas e saberes no enfrentamento de cada situação identificada a fim de propor soluções e intervir de maneira apropriada a cada realidade. Desse modo, emergem as ações educativas como ferramenta indispensável para estimular o autocuidado nos usuários. Objetivo: Relatar a experiência vivenciada durante as atividades de intervenção em educação em saúde realizadas com os profissionais da ESF. Metodologia: Estudo descritivo, tipo relato de experiência. As atividades foram desenvolvidas em uma Unidade de Atenção Primária à Saúde, localizado no município de Quixeré, Estado do Ceará. Resultados: Percebeu-se que a partir da intervenção foi possível despertar momento de reflexão sobre a assistência em saúde, inclusive em relação às percepções de biossegurança, favorecendo assim a troca de saberes. Tal atividade contribuiu ainda, para a construção e o aprimoramento do saber-fazer no tocante a um dos processos de trabalho da enfermagem, o ensinaraprender. Conclusões: A Educação em Saúde propõe-se a contemplar os princípios do Sistema Único de Saúde por intermédio da promoção da saúde, da conscientização do indivíduo e da comunidade com o objetivo de despertar a autonomia dos sujeitos.

Palavras-Chave: Educação em Saúde; Pessoal de Saúde; Estratégia Saúde da Família.

\section{ABSTRACT}

Introduction: The Family Health Strategy is found by multiprofessional teams that must act in an interdisciplinary perspective, articulating their practices and knowledge in facing each situation identified for proportions, solutions and to intervene in an appropriate manner to each reality. In this way, it emerges as educational actions as an indispensable tool to encourage self-care in users. Objective: to report an experience lived during the intervention activities in health education carried out with the professionals of the Family Health Strategy. Methodology: Descriptive study, type of experience report. The activities were carried out in a Primary Health Care Unit, located in the municipality of Quixeré, state of the Ceará. Results: It was realized that from the intervention it was possible to awaken a moment of reflection on health care, including in relation to the perceptions of biosafety, thus favoring the exchange of knowledge. Such activity also contributed to the construction and improvement of know-how in relation to one of the nursing work processes, the use-learn. Fonclusions: Health Education proposes to contemplate the principles of the Unified Health System through health promotion, awareness of the individual and the community in order to awaken the subjects' autonomy.

ywords: Health Education; Health Personnel; Family Health Strategy. 


\section{RESUMEN}

Introducción: La Estrategia de Salud Familiar está formada por equipos multidisciplinares que deben actuar en la perspectiva interdisciplinaria, articulando sus prácticas y conocimientos para hacer frente a cada situación identificada para proponer soluciones, e intervenir adecuadamente a cada realidad. Así, las acciones educativas surgen como una herramienta indispensable para estimular el autocuidado en los usuarios. Objetivo: Informar de la experiencia experimentada durante las actividades de intervención en educación sanitaria realizadas con los profesionales de la Estrategia de Salud Familiar. Metodología: Estudio descriptivo, tipo de informe de experiencia. Las actividades se desarrollaron en una Unidad de Atención Primaria de Salud, ubicada en el municipio de Quixeré, Ceará. Resultados: Se observó que a partir de la intervención era posible despertar un momento de reflexión sobre la atención sanitaria, incluso en relación con las percepciones de bioseguridad, favoreciendo así el intercambio de conocimientos. Esta actividad también contribuyó a la construcción y mejora del know-how con respecto a uno de los procesos de trabajo de enfermería, la enseñanza-aprendizaje. Conclusiones: La Educación en Salud pretende contemplar los principios del Sistema Unificado de Salud a través de la promoción de la salud, la conciencia del individuo y de la comunidad con el fin de despertar la autonomía de los sujetos.

Palabras clave: Educación en salud; Personal de salud; Estrategia de salud familiar. 


\section{Introdução}

A Estratégia Saúde da Família (ESF) é constituída por equipes multiprofissionais que devem atuar numa perspectiva interdisciplinar, articulando suas práticas e saberes no enfrentamento de cada situação identificada, a fim de propor soluções e intervir de maneira apropriada ${ }^{1}$. Nesse sentido, os profissionais de saúde trabalham diariamente com pessoas que possuem o seu referencial de vida, que têm os seus valores e crenças estabelecidos, mas, muitas vezes o saber popular e suas vivências não são levados em consideração².

Portanto, para trabalhar num contexto de educação em saúde na prática junto às comunidades, é imprescindível que os profissionais estabeleçam uma relação entre as ciências da saúde, as ciências sociais e a educação, com a finalidade de promover uma ação educativa democrática, respeitando a liberdade individual em busca do processo de conscientização ${ }^{3}$.

A educação em saúde é uma prática centrada na sociedade, contribui para a formação e desenvolvimento da visão crítica das pessoas a respeito de sua saúde, instigando a busca de soluções e a organização da ação coletiva. Além disso, Compartilhar saberes com a finalidade de prevenir, promover e recuperar a saúde por meio de ações educativas, possibilita ao indivíduo um saber coletivo que reflete em sua autonomia e na emancipação para o cuidar de si, da família e do meio social ${ }^{4}$.

Ainda que a educação em saúde seja um dos instrumentos da promoção, tal prática tem sido insuficiente no desenvolvimento no sistema de saúde, destacando-se a necessidade de os profissionais dessa área receber educação permanente e incluir novas possibilidades metodológicas de atuação ${ }^{5}$.

Desse modo, dentre as ações da ESF, emergem as ações educativas como ferramenta fundamental para estimular tanto o autocuidado, como a autoestima dos indivíduo. E muito mais que isso, de toda a família e comunidade, promovendo reflexões que conduzam a modificações nas atitudes e condutas dos usuários ${ }^{6}$.

Tendo em vista que a educação envolve também a responsabilização da opulação sobre seus hábitos e estilos de vida, destaca-se a importância da fermagem como profissão de compromisso social, sensível aos problemas e direitos hanos, e como ciência que busca novas metodologias para o alcance da melhoria 
da qualidade de vida e da assistência, mediante a execução de atividades educativas em saúde ${ }^{7}$. Contudo, a prática da educação em saúde requer do profissional análise crítica de sua atuação, pois serve de instrumento para construção da participação popular nos serviços de saúde e para o aprofundamento da intervenção da ciência no cotidiano das famílias e sociedades ${ }^{8}$.

Nesse contexto, a enfermagem assume importante papel na consolidação de uma sociedade mais justa e democrática, pois, mediante o cuidado, ela tem a oportunidade de educar o outro para a saúde ${ }^{4}$, uma vez que a enfermagem é uma profissão dedicada ao cuidado humano e tem a educação em saúde como um importante eixo norteador para o desenvolvimento de sua prática9 ${ }^{9}$ Visto que o trabalho destes dois elementos: cuidar e educar, de maneira articulada é o caminho para o exercício de uma prática profissional renovada, que reúna saberes de forma criativa, ética, política e técnico-científica ${ }^{10}$.

Contudo, o processo de intervenção justifica-se pela relevância de intervir positivamente no contexto em que a comunidade e os profissionais estão inseridos, realizando ações de educação em saúde voltadas para a prevenção não somente das hepatites virais, mas sim ampliadas a problemas decorrentes dos processos de trabalho, e destacando a importância do trabalho da equipe saúde da família enquanto multiplicadores de informações.

Portanto, o estudo objetiva relatar a experiência vivenciada durante as atividades de intervenção em educação em saúde realizadas com os profissionais da ESF.

\section{Metodologia}

Trata-se de um estudo descritivo, tipo relato de experiência, elaborado com profissionais da saúde no contexto da pesquisa participante. Implica dizer que a mesma está associada necessariamente na participação tanto do pesquisador no ntexto, grupo ou cultura que está a estudar, quanto dos sujeitos que estão envolvidos processo da pesquisa. Ademais, tem como objetivo principal a intervenção na 
realidade da produção dos serviços, a partir do desenvolvimento de práticas de educação em saúde na Atenção Primária à Saúde.

Refere-se a um projeto de educação em saúde desenvolvido por quatro alunas do curso de enfermagem da Universidade Potiguar - UnP. A ação foi realizada a partir de uma atividade disparada pela Universidade, na qual o tema abordado foi selecionado a partir de demandas elencadas pelas discentes durante a captação da realidade em meio a visita à Unidade de Saúde Sede II localizada na cidade de Quixeré-CE, pois foi observada a ausência de exposição e comunicação do tema "Hepatites Virais" entre a comunidade e os profissionais de saúde.

Portanto, a base metodológica utilizada na atividade foi a Teoria de Intervenção Práxica da Enfermagem em Saúde Coletiva (TIPESC), que aplica a sistematização da dinâmica de captar e interpretar um fenômeno articulado aos processos de produção e reprodução sociais referentes ao processo saúde-doença de uma determinada coletividade, no marco de sua conjuntura e estrutura, dentro de um contexto social historicamente determinado ${ }^{11}$.

Para divulgação da atividade, foi disponibilizado previamente cartazes informativos contendo data, hora e o tema a ser discutido.

Todos os profissionais da Unidade de Saúde Sede II foram convidados para participar dessa atividade. Os profissionais Auxiliares de Serviços Gerais (ASG) não tiveram disponibilidade por estarem exercendo suas funções na unidade. Contudo, um total de dez profissionais de saúde participaram, sendo eles o médico, a enfermeira, dois técnicos de enfermagem e seis Agente Comunitários de Saúde (ACS).

As atividades foram desenvolvidas no dia 26/05/2017, numa sexta-feira, pois geralmente é nesse dia da semana que os trabalhadores se reúnem para planejar suas atividades.

A atividade foi iniciada com uma dinâmica quebra-gelo para uma melhor interação entre as alunas e os profissionais de saúde. Logo após, foi realizada a xposição do tema "Contextualizando as Hepatites Virais", onde os trabalhadores espondiam perguntas sobre as hepatites virais através de placas ilustrativas, uma ntendo Mito e a outra Verdade. 
Após, foi dado prosseguimento as discussões sobre o tema a partir de conhecimentos adquiridos naquela intervenção, bem como também conhecimentos prévios que os profissionais já possuíam.

Para finalizar o momento, foi distribuído lembrança simbólica do evento e servido um coffee break para promover a confraternização dos envolvidos.

\section{Resultados e Discussão}

Diante desse cenário, tornou-se notória a carência de ações de educação em saúde voltada aos profissionais dessa unidade, evidenciada mediante a solicitação dessas pelos mesmos, temas que versem sobre conteúdos bastante discutidos, principalmente a partir da realidade da unidade. Observou-se também a fragilidade de ações e estratégias com foco na educação em saúde relacionada à temáticas de interesse da população adscrita por esses profissionais.

Desse modo, a Educação em Saúde é um instrumento que tem como objetivo empoderar sujeitos que, por meio do diálogo, coloque-os no centro do processo de construção do conhecimento ${ }^{11}$. Com o desenvolvimento da intervenção foi possível viabilizar aos profissionais da ESF um espaço para discussão e reflexão sobre a educação em saúde, evidenciando a importância da educação permanente no que tange os processos de trabalho. Logo, espera-se que os trabalhadores possam compartilhar os seus conhecimentos adquiridos durante ações educativas com os usuários do território adscrito, numa perspectiva de promoção à saúde.

Essas práticas educativas compõem a prática social da enfermagem e caracterizam-se como instrumentos valiosos no processo de trabalho em saúde. Estando a educação em saúde presente no processo de trabalho e no ato de cuidar do enfermeiro, a participação desse profissional é de suma importância na organização e desenvolvimento das ações ${ }^{12}$.

Nesse contexto, vale ressaltar que o enfermeiro é considerado um importante ducador nesse processo, pois além de organizador desses momentos, eles acabam ecutando também a educação em saúde com maior ênfase nos momentos da nsulta de enfermagem. 
Notou-se também a não efetivação de ações de Educação Permanente em Saúde (EPS). Este é um instrumento estratégico e indispensável para a gestão do trabalho e da educação na saúde, porque sua ideologia é baseada na aproximação entre profissionais e pacientes, considerando as particularidades dos lugares em que estes vivem. Nesse processo, o "ouvir" do profissional é mais importante do que o seu "falar"13.

No entanto, tem sido um grande desafio qualificar trabalhadores da ESF a partir do arcabouço da educação permanente, pois, há simultaneamente a disputa pela atualização corriqueira de conhecimentos e práticas, onde insere-se em uma explícita construção das relações e dos processos que advém intrinsecamente das equipes ${ }^{14}$.

Além disso, a discussão se ampliou em relação à biossegurança dos próprios profissionais diante às diversas doenças. Fazendo com que despertasse um olhar em relação ao autocuidado dos próprios profissionais de saúde, por estarem constantemente expostos a riscos.

Percebeu-se que, apesar do conhecimento que os profissionais da ESF tinham sobre as hepatites, os mesmos encontraram-se aptos adquirirem mais conhecimento, a fim de estarem aptos a tomarem medidas de precaução, tanto individual como coletivo, promovendo atitudes preventivos contra diversas doenças.

No entanto, um dos objetivos para o desenvolvimento da intervenção, era atingir todas as categorias profissionais, tendo como limitação a ausência das ASG por estarem desenvolvendo suas atividades no momento da intervenção. Assim, para um próximo encontro foi sugerido que o mesmo tema fosse trabalhado com os usuários e as ASG da unidade, a partir da criação de uma sala de espera, onde espera-se que o projeto venha a ter uma continuidade de pelo menos uma vez por mês.

Com isso, percebeu-se a relevância de trabalhar ações de educação em saúde na ESF, no horizonte da promoção à saúde, pois esta favorece a construção de ações que podem responder de forma efetiva às demandas e necessidades de saúde da pessoa, família e coletividade, numa perspectiva de produzir saúde, articulando o modo de ensar e operar com as demais políticas e tecnologias ${ }^{15}$. Dessa forma, poderá contribuir para o fortalecendo dos processos de conscientização e autonomia dos sujeitos e upos sociais com o intuito de propiciar mudanças nos hábitos de vida. 
Assim sendo, poderá favorecer o fortalecimento do relacionamento entre profissionais e usuários, a partir da criação de vínculos, enfatizando a importância de momentos de construção de espaços que possibilitem a troca de saberes, de interação e aprendizado, de maneira que qualifique principalmente os processos de promoção, prevenção e longitudinalidade do cuidado.

\section{Conclusões}

A Educação em Saúde propõe-se a contemplar princípios do Sistema Único de Saúde (SUS) por intermédio, em especial, das ações de promoção e prevenção da saúde. Neste sentido, o recorre da intervenção possibilitou aos discentes uma nova experiência no campo da Atenção Primária à Saúde, uma vez que possibilitou a aproximação com os profissionais de saúde, cenário ainda não explorado pelas discentes, durante a vida acadêmica. Nesse sentido, foi possível perceber a multiplicidade de espaços e públicos passíveis de intervenções educativas dessa natureza.

Tal atividade contribuiu, ainda, para a construção e o aprimoramento do saberfazer da enfermagem no tocante a um dos seus processos de trabalho, o ensinaraprender, pois aponta princípios para a organização de uma educação profissional ampliada, de forma que considere as especificidades das diversas unidades de produção do cuidado em saúde.

Por fim, constatou-se que a vivência propiciou aos acadêmicos de enfermagem uma nova forma de trabalhar Educação em Saúde, a partir das práticas realizadas com os multiprofissionais, no sentido de vislumbrar caminhos e cenários de atuação capazes de renovar o exercício do processo educativo em saúde, tendo em vista a superação de práticas pontuais e lineares de cuidado.

\section{eferências}

Pedrosa JIS, Teles JBM. Consenso e diferenças entre equipes do Programa de Saúde ha Família. Revista de Saúde Pública. 2001; 35: 303-311. 
2. Budó MLD, Saupe R. Conhecimentos populares e educação em saúde na formação do enfermeiro. Revista Brasileira Enfermagem. 2004; 57(2): 165-169.

3. Pessanha RV, Cunha FTS. A aprendizagem-trabalho e as tecnologias de saúde na Estratégia Saúde da Família. Texto Contexto Enfermagem [online]. 2009; 18(2): 233240.

4. Negri B. Saúde boa e vida melhor para 50 milhões. Revista brasileira de Saúde Família. 2002.

5. Salci MA, Maceno P, Rozza SG, Silva DMGV, Boehs AE, Heidemann ITSB. Educação em saúde e suas perspectivas teóricas: algumas reflexões. Texto Contexto Enferm, Florianópolis. 2013; 22(1): 224-230.

6. Machado MFAS, Monteiro EMLM, Queiroz DT, Vieira NFC, Barroso MGT. Integralidade, formação de saúde, educação em saúde e as propostas do SUS - uma revisão conceitual. Ciência Saúde Coletiva. 2007; 12: 335-342.

7. Lopes EM, Anjos SJSB, Pinheiro AKB. Tendência das ações de educação em saúde realizadas por enfermeiros no brasil. Revista de Enfermagem UERJ. 2009; 273-277.

8. Progianti JM, Costa RF. Práticas educativas desenvolvidas por enfermeiras: repercussões sobre vivências de mulheres na gestação e no parto. Revista Brasileira de Enfermagem. 2012; 65(2): 257-263.

9. Fava SMCL, Nunes ZB, Gonçalves MFC, Nogueira MS. Educação em saúde e adesão ao tratamento na perspectiva histórico cultural. Sau \& Transf Soc. 2011; 2(1): 81-87.

10. Brandão-Neto W, Silva ARS, Monteiro EMLM, Freitas CMSM, França ISX, Medeiros CCM. Educação em saúde como ferramenta do cuidado de enfermagem: revisão integrativa da literatura. Revista de Enfermagem UFPE on Line. 2011; 1541-1548.

11. Egry EY. Saúde coletiva: construindo um novo método em enfermagem. São Paulo (SP): Ícone; 1996; 144-144.

12. Andrade ACV, Schwalm MT, Ceretta LB, Dagostin VS, Soratto MT. Planejamento das ações educativas pela equipe multiprofissional da Estratégia Saúde da Família. O Mundo da Saúde, São Paulo. 2013; 37(4): 439-449.

Maia JDS, Silva AB, Melo RHV, Rodrigues MP, Junior AM. A educação em saúde para usuários hipertensos: percepções de profissionais da estratégia saúde da família. Revista Ciência Plural. 2018; 4(1): 81-97. 
14. Medeiros LCM. Educação Permanente como instrumento de mudança na rede de atenção à saúde com foco na Estratégia Saúde da Família: um relato de experiência. Revista Ciência Plural. 2015; 1(1): 65-74.

15. Silva MIL. Promoção da saúde ao portador de diabetes mellitus na atenção primária à saúde no município de Nova Friburgo. Universidade Federal Fluminense (UFF): Instituto de Saúde Coletiva - Programa de Pós-Graduação em Saúde Coletiva; 2020. 